\section{More research won't crack misconduct}

The US National Academy of Sciences has issued 5 reports in the past 28 years on research misconduct and detrimental research practices. Each concluded with a strikingly similar set of recommendations.

In 1989, for example, we were advised of "a need for additional research to clarify the basic factors that influence professional conduct". The panel of 2002 found that "existing data are insufficient to enable [the committee] to draw definitive conclusions as to which elements of the research environment promote integrity". And in 2017, the panel report called for government agencies and private foundations to "fund research to quantify, and develop responses to, conditions ... linked to research misconduct".

In our view, however, the root causes and potential remedies are evident. Ignorance of good research practices is now addressed by mandatory training. Carelessness can be reduced by conscientious mentoring. Fear of failure requires a shift in academia’s reward system. Preventing bias requires researchers to have heightened self-awareness and a boost in conscience. And cheaters need to fear detection: they must face meaningful penalties while whistle-blowers remain protected.

Why, then, does the academy repeatedly call for further research when potentially effective remedies are available and yet to be implemented?

Donald S. Kornfeld Columbia University, New York City, USA. Sandra L. Titus Northfield, Minnesota, USA. dsk3@cumc.columbia.edu

\section{GM-food regulations: engage the public}

Your call to harmonize rules for genetically modified (GM) animals and plants (Nature 546, 327-328; 2017) echoes scientists' pleas to modernize the 1986 Coordinated Framework for the Regulation of Biotechnology. The framework grants jurisdiction over biotechnology products to US federal agencies, including the Food and Drug Administration (FDA). Yet urging researchers to scrutinize definitions and look for legal loopholes is impracticable. Increasing public education and engagement of the scientific issues concerning GM food should be researchers' main focus.

The importance of public engagement was illustrated decades ago with the use of recombinant bovine growth hormone in dairy cattle. The practice sparked widespread speculation about its safety and prompted the FDA's unprecedented decision to publish health and safety data ahead of formal approval, in efforts to allay public concerns (J. C. Juskevich and C. G. Guyer Science 249, 875-884; 1990). The decision applied only to that case, but may become relevant in the future.

Policymakers should consider the growth-hormone case when outlining new boundaries for data disclosure and regulatory exemptions applicable to geneedited products. Regulations must take into account the interests of GM-product developers to ensure that public disclosures do not undermine intellectual-property rights (see also go.nature.com/2tcoezq). Paul Enríquez North Carolina State University, Raleigh, USA. penriqu@ncsu.edu

\section{GM-food regulations: US agencies respond}

The US Food and Drug Administration (FDA) and the US Department of Agriculture (USDA) have issued documents about genetically modified (GM) animals and plants for public comment (Nature 546, 327-328, 2017). Discussions are ongoing, so your implication that these have "come to opposite conclusions" seems premature.
You note that the agencies are confined by agency-specific statutes, but do not fully acknowledge the differences in the statutes' scope and focus. For example, the FDA has to evaluate the effects of intentionally altered genomic DNA on the health of the modified animal as well as any risk to food safety, whereas the USDA needs to focus on plant health. In addition, the FDA oversees the safety of genetically engineered plants as food for humans and animals, as described in the Coordinated Framework for the Regulation of Biotechnology. Jason Dietz, Laura Epstein FDA, Silver Spring, Maryland, USA. Sidney W. Abel Animal and Plant Health Inspection Service, USDA, Riverdale, Maryland, USA. jason.dietz@fda.hhs.gov

\section{BaBar Collaboration first to see anomaly}

Your discussion on the mysteries of $B$ meson particles refers to the ' $B$ factories' where these are being studied (Nature 546, 185-186; 2017). Aside from CERN's Large Hadron Collider (LHCb) in Geneva, Switzerland, and Belle at Japan's High Energy Accelerator Research Organization in Tsukuba, you should have mentioned the BaBar Collaboration at the SLAC National Accelerator Laboratory in California (go.nature. com/2ubp28p).

The BaBar Collaboration reported the first hint of new fundamental particle physics (see J. P. Lees et al. Phys. Rev. Lett. $109,101802 ; 2012)$. It found a 3.4-sigma deviation from the expectations of the standard model of fundamental particles and their interactions. Subsequent measurements published in 2015 by LHCb and by Belle in 2015-17 confirmed the BaBar result. With these additional data, together with a new 2017 measurement from $\mathrm{LHCb}$, the significance of the deviation from the standard model has grown to 4-sigma.

Tom Browder Belle II
Collaboration, University of Hawaii at Manoa, Honolulu, USA. Giovanni Passaleva $\mathrm{LHCb}$ Collaboration, Istituto Nazionale di Fisica Nucleare, Florence, Italy. Michael Roney BaBar Collaboration, University of Victoria, British Columbia, Canada. mroney@uvic.ca

\section{Improve contactless sensing technology}

Millions of Hall devices are manufactured each year for use in contactless sensing. These sensors are empowering green transport as part of brushless electric motors in aircraft and in electric cars, for example, and are important in anti-lock braking systems and electronic compasses. To expand future applications, their performance needs to be improved.

Today's Hall devices can be rendered ineffective for sensing by an offset voltage problem. This is a spurious voltage caused by factors such as contact misalignment - that appears across the Hall contacts even in the absence of a magnetic field. It has long been known that a sign-reversed Hall voltage can be produced when a Hall device that contains an interior void is turned inside-out with respect to the magnetic field (R. G. Mani and K. von Klitzing Appl. Phys. Lett. 64, 1262-1264; 1994; see also M. Briane and G. Milton Arch. Rational Mech. Anal. 193, 715-736; 2009 and C. Kern et al. Phys. Rev. Lett. 118, 016601; 2017). This phenomenon is useful for engineering the distribution of current so as to reduce offset voltages, increasing magneticfield sensitivity.

An industrial partner will be needed to integrate these concepts into the technology of silicon electronics to realize a better, low-cost, smart Hall sensor for the mass market. Ramesh G. Mani, Annika Kriisa Georgia State University, Atlanta, USA.

mani.rg@gmail.com 